General Conference (Part A)

\title{
MULTILEVEL RS/CONVOLUTIONAL CONCATENATED CODED QAM FOR HYBRID IBOC-AM BROADCASTING
}

\author{
S.-Y. Chung ${ }^{1}$ and H. Lou \\ Massachusetts Institute of Technology \\ Cambridge, MA 02139. \\ Lucent Technologies Bell Labs \\ Murray Hill, NJ 07974.
}

\begin{abstract}
Bandwidth efficient modulation schemes using ReedSolomon (RS) codes are proposed for Hybrid In-BandOn-Channel (IBOC) systems that broadcast digital audio signals simultaneously with analog Amplitude Modulation (AM) programs in the AM band. Since both the power and bandwidth allocated for digital audio transmission are limited in this application, the system cannot afford to add enough redundancy for error control using conventional concatenated coding schemes. We show that by using multilevel RS and convolutional concatenated coded Quadrature Amplitude Modulation (QAM), efficient modulation schemes can be obtained for applications such as IBOC-AM broadcasting.
\end{abstract}

\section{INTRODUCTION}

Hybrid IBOC systems $[1 ; 2]$ are used in the AM band $[1 ; 3]$ to broadcast digital audio signals simultaneously with analog AM programs. In these systems, RS codes are used for error protection of the digital information. RS codes are non-binary codes that are widely used because of their good distance properties and efficient decoding algorithms [4]. RS codes have a wide range of code rates and can correct burst errors that are prevalent in the AM channels in the presence of interference. A RS decoder also provides an error flag that indicates the reliability of the decoded frame. Such a flag is essential for the error mitigation or concealment algorithms that sophisticated source decoders provide.

Multilevel coding combined with multistage decoding first proposed by Imai and Hirakawa [5] uses several block codes to construct a good coding scheme for band width limited channels. Sayegh [6] used block codes at each level and showed that it is possible to achieve coding gain of about $3 \sim 7 \mathrm{~dB}$ with simple block codes such as repetition codes and Bose-Chaudhuri Hocquenghem $(\mathrm{BCH})$ codes. Husni and Sweeney [7] showed that by using RS codes as component codes for multilevel coding combined with M-ary Phase Shift Keying (MPSK)

${ }^{1}$ Work done during an internship at Lucent. modulation, we can get coding gain of about $1 \mathrm{~dB}$ compared to the non-multilevel schemes.

This paper explores efficient ways of applying RS codes to $2^{m}$-QAM for IBOC systems by using multilevel RS coded QAM - a joint RS coding and modulation scheme that applies a different RS code of appropriate rate to each bit of the m-bit QAM symbol. We found that, for example, for a code rate of 0.8 , by using multilevel RS coded QAM, one can get a coding gain of about $4 \mathrm{~dB}$ when the Bit-Error-Rate (BER) is $10^{-7}$. To extend the idea further, we found that by using a concatenated coding scheme for the critical levels in the multilevel coding scheme, an additional $0.8 \mathrm{~dB}$ coding gain can be obtained. This multilevel concatenated RS/convolutional coded QAM scheme falls into the general structure of the multilevel partition codes described in [8].

In the following sections, we will first describe the conventional non-partitioned mapping scheme in Section II.A. After that, a two-level RS coded modulation scheme is given in Section II.B, a general multilevel RS coded modulation scheme is given in Section II.C and the multilevel concatenated RS and convolutional coded QAM scheme is given in Section II.D. We will also compare the performances of these different schemes in Section III.

\section{RS CODED QAM SCHEMES}

\section{A. Non-partitioned mapping of RS symbols to $Q A M$} symbols

To map a RS coded symbol into a QAM constellation point, the current techniques use RS codes based on $\operatorname{GF}\left(2^{m}\right)$ if the modulation is $2^{m}$-QAM [9]. Thus, each $m$-bit RS symbol can map directly into an $m$-bit QAM symbol. However, this limits the choice of RS codes one can apply and thus may affect the error-correction capability. For example, if a 32-QAM system is used, a perfect matched RS code will be based on $\operatorname{GF}\left(2^{5}\right)^{2}$. For a given coding rate of 0.8 , a RS code of $(30,24)$ can

\footnotetext{
${ }^{2}$ Two 32-QAM symbols can also be matched perfectly to one symbol of a RS code based on $G F\left(2^{10}\right)$. However, the decoding complexity will be much higher.
} 
be applied to match the alphabet size. Since longer block codes are desired for more powerful error protection, one may want to use a $(60,48)$ RS code based on $\mathrm{GF}\left(2^{6}\right)$ because it can correct twice as many random errors as the $(30,24)$ code [3]. However, this will result in a mismatch between the 6-bit RS symbols and the 5-bit QAM symbols. One way to match the modem symbols to the RS code alphabet is to take every 6 modem symbols and map them onto 5 RS symbols. With this scheme, shown in Figure 1, one can see that $1 / 3$ of the modem symbols will only affect one RS symbol and $2 / 3$ of the modem symbols will affect at most two RS symbols.

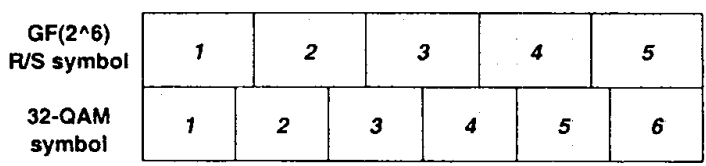

Fig. 1. Mapping rule for 32-QAM, RS GF $\left(2^{6}\right)$ symbols.

\section{B. Two-level RS coded $Q A M$}

The motivation behind using a two-level RS coded QAM scheme is that the least significant bits in QAM symbols are more likely to be corrupted by noise. Thus, for a given amount of redundancy, it may be a good idea to protect only those bits rather than to use RS codes for all bits. In this system, an $(n, k)$ RS code over $\mathrm{GF}\left(2^{q}\right)$ is used in place of the convolutional coder in TCM as shown in Figure 2, where $0<k<n \leq 2^{q}-1$. Since the RS symbol size is $q$ bits, the total number of information bits is $k q$ and the total number of encoded bits is $n q$.

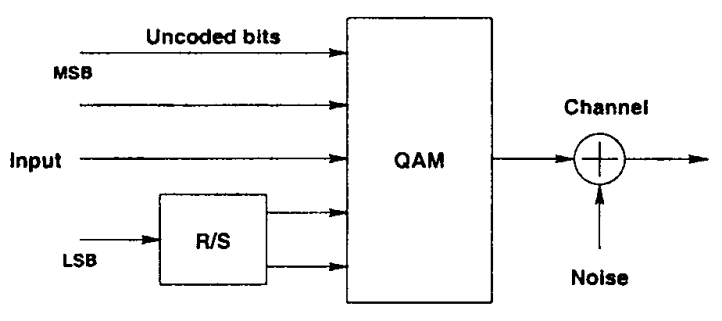

Fig. 2. Encoder for two-level RS coded QAM

Input bits are divided into two groups - one uncoded and the other RS coded. Let $r$ denotes the number of the uncoded bits per two dimensions and let $s$ denotes the number of bits per two dimensions that will be RS coded, thus $(r+s)$ bits are being transmitted. Since the RS encoder takes $k q$ bits, we assume $k q$ is divisible by $s$ and $n q$ is divisible by $t=m-r$, the number of coded bits per two dimensions. The uncoded bits and the coded bits are mapped to QAM symbols by Ungerboeck's mapping by set partitioning [10]. The $2^{m}$-QAM signal constellation is partitioned into two subsets, with each subset having $2^{m-1}$ points and the partitioning is done in a way that the minimum Euclidean distance between points in each subset is maximized. We can do this partitioning iteratively so that after $m$ steps, we have $2^{m}$ single point subsets. At the $t^{\text {th }}$ step, we have $2^{t}$ subsets, where each subset has $2^{m-t}$ points. We use $t$ coded bits as an index for the $2^{t}$ subsets and use the remaining $r$ uncoded bits to choose a point within the subset specified by the $t$ coded bits. The decoder first decodes a sequence of subsets of length $\frac{n q}{t}$ by using hard decision. After that, this $q$-bit sequence of length $n$ is corrected by the RS decoder and this corrected bits are used to generate a corrected sequence of subsets. At the next stage of decoding, the remaining sequence of the $r$-bit symbols is decoded by choosing a point in each subset that is closest to the received point at each time. If the RS decoder cannot correct the errors because there are too many errors, it will not attempt to correct them. Thus, error propagation due to incorrect error corrections to the next stage of decoding is unlikely since the error flag of a RS decoder is very reliable.

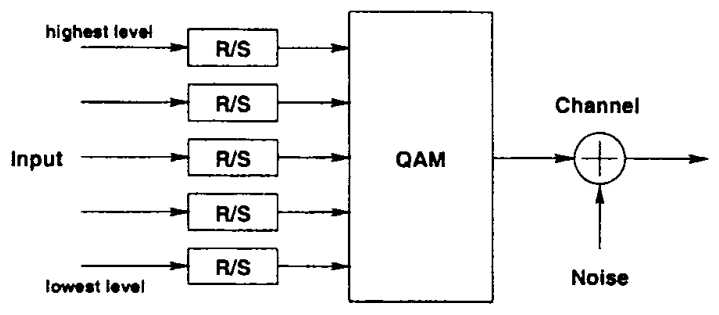

Fig. 3. Encoder for multilevel RS coded QAM.

\section{Multilevel RS coded QAM}

The multilevel RS coded QAM scheme is a generalization of the two-level RS coded QAM scheme described in the previous section. In multilevel RS coded QAM, we construct an encoder with $m$ different $R S$ coders with different rates combined with a $2^{m}$-QAM constellation as shown in Figure 3, where low rate RS codes are used for lower levels more error protection is needed.

Let $\left(k_{0}, k_{1}, \ldots, k_{m-1}\right)$ be the set of information symbol sizes for the $m$-level RS encoders, with $k_{0}$ being the information symbol size for the lowest level and $k_{m-1}$ being that of the highest level. The RS codes for each level are defined over $\operatorname{GF}\left(2^{q}\right)$ and let $n$ be the number 


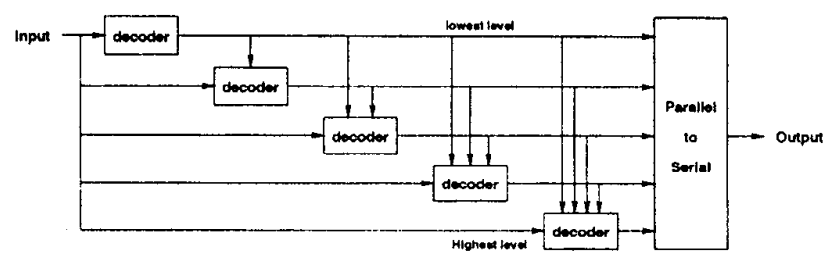

Fig, 4. Decoder for multilevel RS coded QAM.

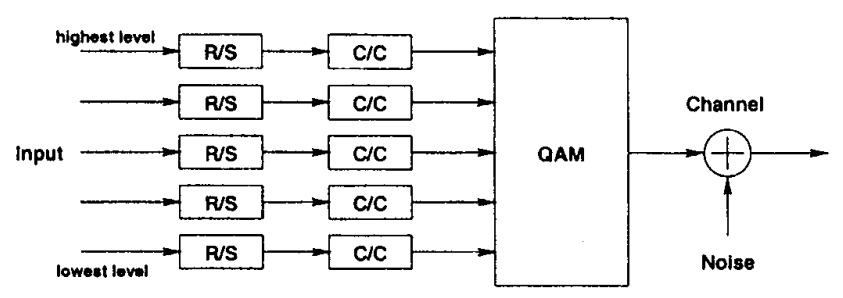

Fig. 5. Encoder for multilevel concatenated RS coded QAM using convolutional codes $(\mathrm{C} / \mathrm{C})$.

of output symbols of each RS encoder. The rate of this encoder is $\frac{k}{n m}$, where $k=\sum_{i=0}^{m-1} k_{i}$ is the total number of information symbols. Since there are $n m q$ bits produced by the encoder, $2^{m}-\mathrm{QAM}$ is used $n q$ times to transmit the coded sequence and at each time, $m$ bits are mapped into an $m$-bit QAM symbol by mapping by set-partitioning.

Decoding is performed similarly as in the two-level RS coded QAM. First, the lowest level is decoded and the corrected sequence is used to decode the next level. This is performed iteratively as shown in Figure 4. At each level, hard decision is used on which most RS decoders are based.

\section{Multilevel concatenated RS/convolutional coded $Q A M$}

Since hard decisions are used in the multilevel RS coded QAM scheme, it is possible to get more coding gain by using soft decision decoding. Soft decision decoding for RS codes, however, is too complex to implement. One alternative is to use concatenated coding scheme using a RS code as an outer code and a convolutional code as an inner code, since soft decision decoding (Viterbi algorithm) is available for the inner code. Instead of concatenating a RS code and a convolutional code, we propose applying the concatenated scheme to each level of a multilevel coding scheme as in Figure 5. We will show that, to achieve good coding gain, the concatenated coding scheme may be necessary only for the lower levels in a multilevel coding scheme. These codes also fall into the general structure of the multilevel partition codes described in [8].
Similar to the multilevel RS coded QAM, we assume $\left(k_{0}, k_{1}, \ldots, k_{m-1}\right)$ is the set of information symbol sizes for the $m$ RS encoders from the lowest to the highest levels, where the RS codes are defined over $\operatorname{GF}\left(2^{q}\right)$. We also let $\left(n_{0}, n_{1}, \ldots, l_{m-1}\right)$ be the set of numbers of output symbols of each RS encoder. Further, let $\left(R_{0}, R_{1}, \ldots, R_{m-1}\right)$ be the set of rates of the convolutional encoders for the m-levels, with $R_{0}$ as the rate for the lowest level and $R_{m-1}$ as the rate for the the highest. In addition, let $n$ be the number of output bits of each convolutional encoder such that $\frac{n_{i} q}{R_{i}}$ is equal to $n$, where $n_{i} q$ is the number of output bits of the RS encoder at the $\mathrm{i}$-th level. The rate of this overall encoder is $\frac{k q}{n m}$, where $k=\sum_{i=0}^{m-1} k_{i}$ is the total number of information symbols. Since there are $n m$ bits produced by the encoder, $2^{m}$-QAM is used $n$ times to transmit the coded sequence where coded $m$-bit symbols are mapped into the QAM symbols via mapping by set-partitioning.

Decoding is performed similarly as in the multilevel RS coded QAM scheme except that soft decisions can be exploited in this case because we can use the Viterbi algorithm to decode the convolutional coded bits [11].

\section{Simulation of PERFormance}

After discussing the four different multilevel coding schemes in the previous sections, we compared the performance of the four schemes by simulations. This section discusses the performance of the four schemes using a 32-QAM constellation and an overall channel coding rate of $4 / 5$. The Shannon limit in $E_{b} / N_{0}$ for this case is about $5.74 \mathrm{~dB}$. The BER result using each scheme is compared to the uncoded case where Gray coded 16QAM is used. Simulation results are shown in Figures $6,7,8,9$, for the four coding schemes.

\section{A. Non-partitioned mapping of $R S$ symbols to $Q A M$ symbols}

Figure 6 shows the results for the non-partitioned RS coded 32-QAM scheme described in Section II.A. A $(30,24)$ RS code over GF $\left(2^{5}\right),(60,48)$ RS code over $\mathrm{GF}\left(2^{6}\right)$, and $(100,80) \mathrm{RS}$ code over $\mathrm{GF}\left(2^{8}\right)$ are used. Even though symbol sizes are not matched for the $(60,48)$ and $(100,80)$ codes, simulation results only confirm that longer block codes are generally better, suggesting the mismatch between RS code and QAM alphabets is not a major problem. Coding gain for the $(100,80)$ code is about a $1 \sim 2 \mathrm{~dB}$ at BER $10^{-5} \sim 10^{-7}$.

\section{B. Two-level RS coded $Q A M$}

When we use a two-level RS coded QAM scheme described in Section II.B, we obtain better coding gains than that of the non-partitioned schemes. A $(30,15)$ 


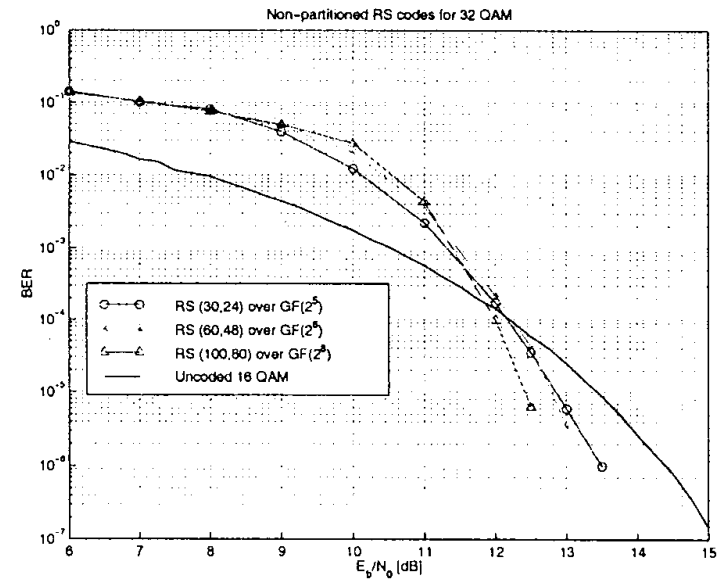

Fig. 6. Simulation results for non-partitioned RS coded QAM.

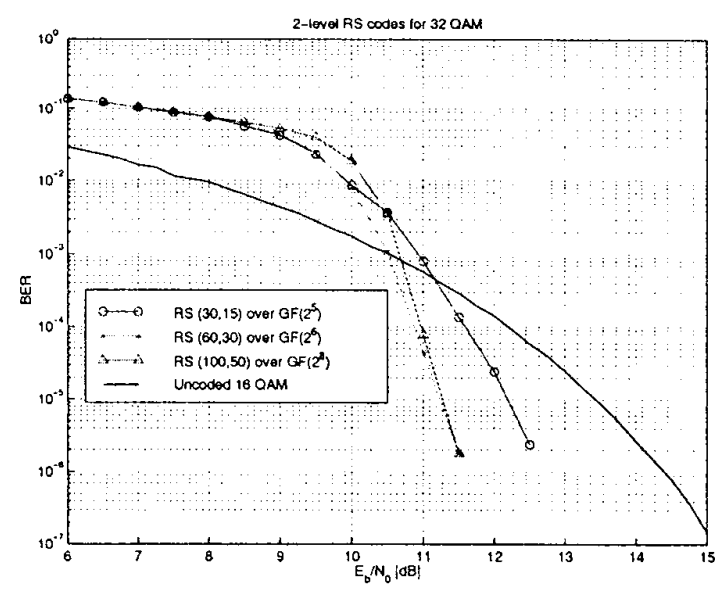

Fig. 7. Simulation results for two-level RS coded QAM.

RS code over $\operatorname{GF}\left(2^{5}\right)$, a $(60,30)$ RS code over $\mathrm{GF}\left(2^{6}\right)$, and a $(100,50)$ RS code over $\mathrm{GF}\left(2^{8}\right)$ are used for this simulation. Among the 5 QAM bits, three bits are uncoded and the other two bits are RS coded. Thus, the overall coding rate is $4 / 5$. Except for the $\mathrm{GF}\left(2^{5}\right)$ case, we observe additional $1.25 \sim 1.75 \mathrm{~dB}$ coding gain compared to that of the non-partitioned case at BER $10^{-6}$ as shown in Figure 7 . $(30,15)$ code performs poorly mainly because it is short but also because the 5-bit RS symbols are not matched to the 2-bit symbol size of the coded QAM bits. When the $(100,50)$ RS code is used, the coding gain is about $3.5 \mathrm{~dB}$ at BER $10^{-7}$.

\section{Multilevel RS coded $Q A M$}

Figure 8 shows the simulation results for the multilevel RS coded 32-QAM scheme (Section II.C) when RS

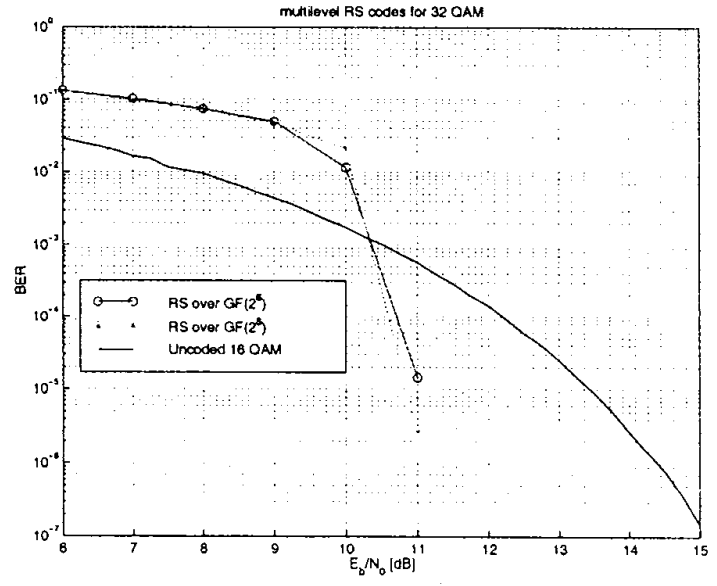

Fig. 8. Simulation results for multilevel RS coded QAM.

codes over $\mathrm{GF}\left(2^{6}\right)$ and RS codes over $\mathrm{GF}\left(2^{8}\right)$ are used. For the $\mathrm{GF}\left(2^{6}\right)$ case, the two highest levels are uncoded and the remaining three levels (from the middle level to the lowest level) are protected by a $(60,58)$ RS code, a $(60,50)$ RS code, and a $(60,12)$ RS code, respectively. For the $\mathrm{GF}\left(2^{8}\right)$ case, two highest levels are also uncoded and the remaining three levels (from the middle level to the lowest level) are protected by a RS $(100,98)$ code, a RS $(100,86)$ code, and a RS $(100,16)$ code, respectively. These code rates are chosen through simulations to minimize BER. When RS codes over $\mathrm{GF}\left(2^{8}\right)$ are used, we get about $4 \mathrm{~dB}$ coding gain at $\mathrm{BER}=10^{-7}$.

\section{Multilevel concatenated RS coded $Q A M$}

Figure 9 shows the performance of the corresponding multilevel concatenated coding scheme where RS codes are used as outer codes and convolutional codes are used as inner codes (Section II.D). A simple rate$1 / 2$ convolutional code with four states is used for the lowest level. Since the rates of the concatenated codes for the other four levels are higher than 0.5 , it is not possible to use a rate- $1 / 2$ convolutional code for those levels. Therefore, for these levels RS codes are used without any convolutional codes. We compare three different schemes using codes over different finite fields. The first scheme uses RS codes over $\mathrm{GF}\left(2^{5}\right)$ and the parameters of the RS codes are $(30,24)$ and $(15,6)$ for the lowest two levels. The highest three levels are not protected and remain uncoded. The second scheme uses RS codes over $\mathrm{GF}\left(2^{8}\right)$ and the parameters (from middle to the lowest level) are $(100,98),(100,86)$, and $(50,16)$, respectively. The higher two levels are uncoded in this scheme. The third scheme uses RS codes over $\mathrm{GF}\left(2^{8}\right)$ 


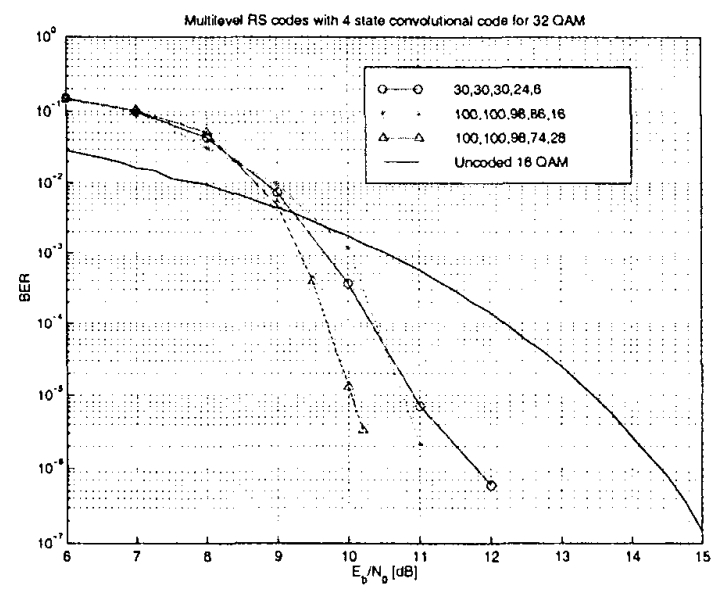

Fig. 9. Simulation results for multilevel concatenated RS coded QAM using a rate-1/2 4-state convolutional code for the lowest level.

and parameters (from middle to the lowest level) are $(100,98),(100,74)$, and $(50,28)$, respectively. The highest two levels are also uncoded as in the second case. We observe that there is about a $4.8 \mathrm{~dB}$ coding gain at $\mathrm{BER} \sim 10^{-7}$ with the third scheme, which is about $0.8 \mathrm{~dB}$ better than that of the multilevel RS coded QAM scheme in Section II.C. The difference in the performance between the second and the third schemes suggests that the code rates for different levels should be optimized for better performance. As in the multilevel RS case without concatenation, the rates for the third scheme are chosen through simulations to minimize BER.

\section{CONCLUSIONS}

Simulation results for $\mathrm{RS}$ coded modulation schemes show that coding gain of $3 \sim 4 \mathrm{~dB}$ can be obtained by using multilevel RS coded QAM combined with multistage decoding and extra coding gain is possible by exploiting multilevel concatenated coding schemes. Without multilevel coding, we can only achieve $1 \sim 2 \mathrm{~dB}$ coding gain when we map RS coded bits directly to a QAM symbol. Multilevel RS coding can achieve an additional coding gain of about $2 \sim 3 \mathrm{~dB}$ by using the same RS encoders and decoders with the same symbol size but with different code rates. In applications where RS codes are required for error protection but when there is not enough redundancy for error protection schemes such as concatenated RS with TCM, these multilevel schemes can be useful, because we can get extra coding gain compared to the non-partitioned RS coded modulation schemes and we can have all the benefits of the RS codes such as error flag and burst er- ror correction capabilities where a reliable error flag is essential for the error mitigation or concealment algorithms that sophisticated source decoders provide and good burst error correction capability is required for IBOC systems in the AM band.

\section{REFERENCES}

[1] C.-E. Sundberg, D. Sinha, H. Lou, P. Kroon, and B.-H. Juang, "Technology advances enabling "inband on-channel" digital sound broadcasting systems," in International Conference on Broadcast Asia, (Singapore), June 1998.

[2] B. W. Kroeger, "Compatibility of FM Hybrid InBand-On-Channel (IBOC) system for digital audio broadcasting," IEEE Transactions on Broadcasting, vol. 43, no. 4, pp. 421-430, 1998.

[3] H. Lou, D. Sinha, and C.-E. W. Sundberg, "Multistream transmission for Hybrid IBOC-AM with embedded/multidescriptive audio coding," Lucent Technologies Bell Labs Technical Memorandum, August 1998.

[4] R. E. Blahut, Principles and Practice of Information Theory. Menlo Park, CA: Addison-Wesley, 1987.

[5] H. Imai and S. Hirakawa, "A new multi-level coding method using error-correcting codes," IEEE Trans. on Information Theory, vol. 23, no. 3, pp. 371-377, 1977.

[6] S. I. Sayegh, "A class of optimum block codes in signal space," IEEE Trans. on Communications, vol. COM-23, no. 10, 1986.

[7] E. Husni and P. Sweeney, "Robust Reed-Solomon coded MPSK modulation," in 6th IMA International Conference Proceedings, (Cirencester, UK), December 1997.

[8] G. J. Pottie and D. P. Taylor, "Multilevel codes based on partitioning," IEEE trans. on Information Theory, vol. 35, pp. 87-98, January 1989.

[9] P. Corbo and J.-C. Belfiore, "Reed-Solomon coded QAM modulation on a Rayleigh fading channel," in PIRMC'92 International Symposium on Personal Indoor and Mobile Radio Communications Proceedings, 1992.

[10] G. Ungerboeck, "Trellis-coded modulation with redundant signal sets: Parts I and II," IEEE Communications Magazine, vol. 25, February 1987.

[11] G. D. Forney, Jr., "The Viterbi algorithm," IEEE Proceedings, vol. 61, pp. 268-278, March 1973. 